\title{
The effect of DNA repair defects on reproductive performance in nucleotide excision repair (NER) mouse models: An epidemiological approach
}

\author{
P.S. Tsai ${ }^{1}$, M. Nielen ${ }^{1}$, G.T.J. van der Horst $^{2}$, B. Colenbrander ${ }^{1}$, J.A.P. Heesterbeek ${ }^{1}$ \\ \& J.M. Fentener van Vlissingen ${ }^{3, *}$ \\ ${ }^{1}$ Department of Farm Animal Health, Faculty of Veterinary Medicine, Utrecht University, P.O. Box 80151, 3508 \\ TD, Utrecht, The Netherlands \\ ${ }^{2}$ Department of Cell Biology and Genetics, Medical Genetic Center, Erasmus University, Medical Center, P.O. Box \\ 1738, 3000 DR, Rotterdam, The Netherlands \\ ${ }^{3}$ Erasmus Laboratory Animal Science Center (EDC), Erasmus University Medical Center, P.O. Box 1738, 3000 \\ DR, Rotterdam, The Netherlands
}

Received 12 April 2005; accepted 5 August 2005

Key words: animal management system, epidemiology, mutant mice, nucleotide excision repair, reproduction

\begin{abstract}
In this study, we used an epidemiological approach to analyze an animal database of DNA repair deficient mice on reproductive performance in five Nucleotide Excision Repair (NER) mutant mouse models on a C57BL/6 genetic background, namely CSA, CSB, XPA, XPC [models for the human DNA repair disorders Cockayne Syndrome (CS) and xeroderma pigmentosum (XP), respectively] and mHR23B (not associated with human disease). This approach allowed us to detect and quantify reproductive effects based on a relatively small number of matings. We measured and quantified the scale of the effect between factors that might influence reproductive performance (i.e. age at co-housing, seasons) and reproductive parameters (i.e. litter size and pairing-to-birth interval -'pbi'). Besides, we detected and quantified the differences in reproductive performance between wild type mice and heterozygous/homozygous NER mutant mice. From our analyses, we found impaired reproduction in heterozygous and homozygous knock out mice; in particular, reduced litter size and lengthened pbi was related to the NER mutation-mHR23B, in heterozygous couples, even if they were otherwise phenotypically normal. Heterozygous mHR23B couples produced a 6.6-fold lower number of $\mathrm{mHR} 23 \mathrm{~B}^{-/-}$pups than indicated by Mendelian expectation; other genetic deficiencies studied were not statistically significant from each other or wild type controls. We concluded that careful epidemiological evaluations by analysis of animal database could provide reliable information on reproductive performance and detect deviations that would remain unnoticed without this. Also, some managerial aspects of mouse breeding could be evaluated.
\end{abstract}

\section{Introduction}

Since the introduction of the first transgenic mouse by Palmiter and coworkers in 1982 (Palmiter et al.,

\footnotetext{
*Author for correspondence

E-mail: m.fentener@erasmusmc.nl
}

1982), genetically modified (GM) animal models have become indispensable tools for biomedical research. With GM mouse models, gene functions can be studied in the intact organism and human disorders can be simulated in a standardized way, allowing research approaches that can not be applied to human patients (Grootegoed et al., 1998; Amiram \& Glickman, 2001). The mouse is 
Table 1. Typical parameters of Reproductive Biology for the European House Mouse, Mus musculus, in the Laboratory

\begin{tabular}{ll}
\hline Parameters & Statistics \\
\hline Gestation length $^{\mathrm{a}}$ & $19-20$ days \\
Age at weaning $^{\mathrm{a}}$ & 3 weeks \\
Age at sexual maturity $^{\mathrm{a}}$ & 6 weeks \\
Approximate weight $^{\mathrm{a}}$ & at birth: $1 \mathrm{~g}$ \\
& at weaning: $8-12 \mathrm{~g}$ \\
Life span in laboratory $^{\mathrm{a}}$ & adult: $30-40 \mathrm{~g}$ (male $>$ female) \\
Average litter size at birth $^{\mathrm{b}}$ & $1.5-2.5$ years \\
Total number of litters per breeding female & $6-8$ pups/litter \\
\hline
\end{tabular}

Table adapted from handbook: Manipulating the Mouse Embryo - A Laboratory Manual, Second Edition (Hogan et al., 1994). ${ }^{a}$ Parameters varied between different inbred strains. Details can be found in Lyon and Searle (1989) and Festing (1993).

${ }^{\mathrm{b}}$ Litter size at birth depends on many factors, e.g. age of father or mother at paring, prenatal mortality, different strains (reflecting genetic factors such as efficiency of placentation), environmental conditions (Boshier, 1968).

attractive because the animal is small, many inbred and outbred strains with well defined characteristics are available, and it normally has a high reproductive capacity and relatively short generation time (Hogan et al., 1994; O.E.C.D., 1998; Beynen \& Hau, 2001; Van Zutphen et al., 2001). In addition, the mouse can act as a very good tool for genetic studies because a lot of techniques for genetic modification in the germ line such as knock in and knock out techniques are available for this species. Typical parameters of mouse reproductive biology are shown in Table 1. These reproductive parameters vary between inbred strains, e.g. average litter size at birth can vary from 6 to 8 pups; gestation length may vary between 19 and 20 days (Festing, 1993). Genetic modifications may result in reduced reproductive performance, which could either be the direct phenotypic consequence of the genetic alteration (e.g. mutations, deletions) on reproductive physiology, or originate from decreased vitality, behavioral abnormalities or affected health (Simpson, 1998; Van der Meer et al., 2001; de Boer et al., 2002). Therefore, systematic (epidemiological) evaluation of these GM mouse colonies and animal models is necessary (1) to detect minor defects in reproductive capacity (often overlooked in initial analysis of GM mouse phenotypes), (2) to improve colony management, (3) to design more precise experiments (for example, with power analysis one can estimate the minimal or adequate number of samples required to detect differences between groups), and (4) to monitor welfare conditions.
DNA repair mechanisms are highly conserved in eukaryotic species and bulwark the genome from many harmful damages by mutagenesis which may also lead to carcinogenesis (de Boer \& Hoeijmakers, 1999; Lehmann, 2002). So far, besides mHR23B mutation, most of the findings or clinical signs for these mutations in mice did not point toward reproductive problems (Table 2). Some studies revealed a role for ubiquitin in gametogenesis, which caused reduced reproductive performance in female mice and sterility in male mice (Baarends et al., 1999; Ng et al., 2002), but the mechanisms by which mutations of the gene encoding for this protein affect reproductive performance on the female side are still unclear.

Nucleotide excision repair (NER), one of the DNA repair systems, guards the DNA from damage that is caused by exposure to harmful exogenous agents (e.g. oxidative stress, UV light, ionizing radiation) and reactive compounds produced by cellular metabolism (de Boer \& Hoeijmakers, 2000). In man, the clinical consequences of defective NER are dramatically illustrated by the phenotype of rare, autosomal recessive disorders such as Xeroderma pigmentosum (XP) and Cockayne syndrome (CS) (reviewed by Bootsma et al., 2001). Xeroderma pigmentosum (7 complementation groups: XPA through XPG) is characterized by hypersensitivity to solar (UV) light, resulting in pigmentation anomalies and a 2000-fold elevated risk of developing skin cancer in sun-exposed areas of the body, often in combination with progressive neurological 
degeneration (Berneburg \& Krutmann, 2003). Cockayne syndrome patients (2 complementation groups: CSA and CSB) also have a photosensitive skin but have not been reported to develop skin cancer at an increased rate. Instead, CS patients present with postnatal growth failure, impaired sexual development, and severe neurological dysfunction (Nance \& Berry, 1992).

Like in man, XPA and XPC mutations render mouse skin cancer prone (De Vries et al., 1995; Cheo et al., 1996). In contrast to human CS, CSA and CSB animals show a slight cancer predisposition, and only mild CS symptoms such as a reduced body weight, photoreceptor loss and mild neurological impairment (Van der Horst et al., 1997; Van der Horst et al., 2002). The mHR23B NER gene is not known to cause a human syndrome, but inactivation of this gene in the mouse causes morphological abnormalities of the reproductive system in GM mice ( $\mathrm{Ng}$ et al., 2002). The mechanisms of the effects as well as the associations of these NER mutations on reproductive performance in mice remain to be elucidated.

In this study, we used epidemiological tools to explore and evaluate the reproductive performance of these GM mice as part of a large mutant mouse colony. All data related to individual mouse models were present in an Animal Management System (AMS) (www.eur.nl/fgg/ch1/ams). The AMS was designed in house for animal management, including monitoring of clinical signs and discomfort. The AMS is a web-based mySql database that can be accessed by authorized persons through the network. The database included hundreds of genotypes, and acquired data on between 10,000 and 20,000 litters and some 40,000 animals on test annually. Detailed information of the AMS database is described in the Appendix. Using epidemiological approaches, data from an AMS may uncover subtle phenotypic abnormalities that are not immediately evident in one or several individual animals in GM mouse strains and populations. Besides detecting less obvious phenotypic abnormalities, it proved also possible to quantify statistical associations between potential risk factors and reproductive performance.

In the present study, we used epidemiological and statistical approaches to assess the effects of NER mutations on reproductive performance in 
GM mice as well as the scale of the effect by comparing heterozygous and homozygous mutant mice with wild type mice and by comparisons among heterozygous and/or homozygous genotypes.

\section{Materials and methods}

We analyzed AMS breeding records of a NER mutant mouse colony, including heterozygous (genotype as $+/-$ ) and homozygous (genotype as $-/-$ ) mutant mice, for (1) general relations between factors that might influence reproductive performance (i.e. age at co-housing, season and breeding status) and reproductive parameters (i.e. pregnancy rate, litter size and pairing-to-birth interval -'pbi'), (2) the effects of the DNA deficiency on reproductive capacity in GM mice, especially on pregnancy rate, litter size and pbi. Although a couple of reports have appeared in literature (e.g. Grootegoed et al., 1998; Simpson, 1998; Baarends et al., 1999; Peters et al., 2002), relatively few studies have ever evaluated these effects.

Mice were between 2 to 9 months of age and were housed under the same living conditions (1-5 mature mice per cage), with room temperature maintained at $20-22^{\circ} \mathrm{C}, 55-60 \%$ relative humidity, and a light-dark cycle of $12 \mathrm{~h}$ artificial light $/ 12 \mathrm{~h}$ darkness. Animals were fed ad libitum with irradiated Special Diets Services (SDS) 'rat and mouse breeder and grower' autoclaveable pellet diet-CRM (P), product code 801722 (SDS, Witham, Essex, England). Water was acidified to $\mathrm{PH}$ 2.5-3.0 with diluted $\mathrm{HCl}$ and supplied via drinking bottles.

A total of 273 litters from 304 breeding pairs of mice from five NER mutant mouse models, and corresponding controls, in a $\mathrm{C} 57 \mathrm{BL} / 6$ genetic background (backcross generation $>10$ ) were investigated, namely Cockayne syndrome A (CSA) (Van der Horst et al., 2002) and B (CSB) (Van der Horst et al., 1997) protein, mouse homo$\log$ of $S$. cerevisiae repair protein RAD23B (mHR23B) (Ng et al., 2002), XP group A (XPA) (De Vries et al., 1995) and C (XPC) (Cheo et al., 1996). Information was collected on genotype, cohousing date, age of parents at co-housing, date of parturition, numbers and sex of the offspring in the period from $01 / 01 / 2001$ to $01 / 11 / 2004$. Mice could be present repeatedly in the dataset if allowed to breed more than once (this resulted in the analysis of data from 560 individual mice). Mice were divided in groups using criteria consistent with the aim of the various analyses. Detailed information on grouping criteria and the exact number of litters

Table 3. Criteria for grouping and the numbers of pairings and litters tested in each subgroup test

\begin{tabular}{|c|c|c|c|c|c|}
\hline Grouping criterion & Subgroups & & Number of litters tested & Number of pairings & Percentage litters born ${ }^{\mathrm{d}}$ \\
\hline \multirow[t]{10}{*}{ Parental genotype } & Wild type & & 13 & 18 & 72 \\
\hline & Heterozygous couple ${ }^{a}$ & CSA & 16 & 16 & 100 \\
\hline & & $\mathrm{CSB}$ & 16 & 20 & 80 \\
\hline & & mHR23B & 18 & 18 & 100 \\
\hline & & XPA & 31 & 37 & 84 \\
\hline & & $\mathrm{XPC}$ & 20 & 20 & 100 \\
\hline & $\begin{array}{l}\text { Heterozygous in one } \\
\text { gender } \times \text { wild type }\end{array}$ & & 39 & 39 & 100 \\
\hline & Knock out in pair ${ }^{b}$ & $\mathrm{KO}$ male $^{\mathrm{c}}$ & 24 & 25 & 96 \\
\hline & & KO female ${ }^{c}$ & 13 & 14 & 93 \\
\hline & & KO both ${ }^{c}$ & 83 & 97 & 86 \\
\hline Total number & & & 273 & 304 & 90 \\
\hline
\end{tabular}

Aim of the tests: to assess whether litter sizes/pairing to birth intervals are different between Nucleotide Excision Repair mutant strains and/or wild type.

${ }^{a}$ Heterozygous couple: $+/-x+/-$.

'The mHR23B mutants could only be bred from heterozygous couples and are thus not represented in the category 'knock out in pair'. As no significant differences on either litter size or pbi between other mouse models (CSA, CSB, XPA, XPC, knock out in pair) were detected, these data were pooled to increase the number of pairs for tests.

${ }^{\mathrm{c}} \mathrm{KO}$ male: $-/-x+1+,-/-x+/-$; KO female: $+/+\times-/-,+/-x-/-$; KO both: $-/-x-/-$.

${ }^{\mathrm{d}}$ The percentage of litters born reflects the 'pregnancy rate' for each subgroup. 
tested for each subgroup are presented in Table 3. All comparisons were originally designed to be between control $(+/+x+/+)$, heterozygous $(+/-x+/-)$ and homozygous knockout (KO) $(-/-\times-/-)$ matings. Mice used as control group in this study were kept and bred under the same conditions and included both wild type mice by pedigree (genotype as 'wt', 2 pairs), and 'wild type' mice derived from breeding with heterozygous animals that did not carry the modified gene (genotype as ' $+/+$ ', 16 pairs).

Definitions for parameters throughout the study were based on the AMS data as follows: (1) Pregnancy rate: defined as the numbers of successful pregnancies per subgroup divided by the total numbers of mating per subgroups; (2) Litter size: defined as the number of pups counted within $24 \mathrm{~h}$ after birth, both alive and dead; (3) pbi: defined as the duration from co-housing date to parturition date in days; (4) Seasons of cohousing date: Spring (March, April, May), Summer (June, July, August), Autumn (September, October, November), Winter (December, January, February). Additionally, on biological grounds, levels were defined for (1) age at co-housing, young adult ( $\leq 90$ days), adult (90-240 days) or older ( $>240$ days); (2) breeding status, first time and multiparous breeders and on genetic grounds: (3) parental genotype, heterozygous couple: $+/-x+/-$; heterozygous in one gender: included heterozygous in male: $+\mid-x+1+$ and in female: $+/+x+/-$; KO male: $-/-x+/+,-/-x+/-$; KO female: $+/+x-/-,+/-x-/-$; KO both: $-/-\times-/-($ Table 3$)$.

\section{Epidemiological and statistical analysis}

An epidemiological approach was followed to analyze the reproductive data derived from a breeding population, in the absence of an experimental design to study reproductive characteristics specifically.

Statistical analyses were carried out in SPSS 10.0 (Instructional, Research and Client Services (IRCS), USA, http://www.spss.com) and S-PLUS 6 (Insightful Corporation, USA, http://www.s-plus. com). Statistical significance levels for all parameters were set at $p \leq 0.05$. If differences were not statistically significant between groups, data from subgroups were pooled to increase the sample size. For two group comparisons, the $\chi^{2}$-test or Fisher's exact test was used for proportions and independent-samples $T$-test or Mann-Whitney $U$-test was used for continuous variables (Altman, 1991). ANOVA or Kruskal-Wallis $H$-test (Altman, 1991) was followed by multiple comparisons by either Post Hoc tests, Mann-Whitney $U$-test with Bonferroni correction (Altman, 1991) or by nonparametric multiple comparisons tests (Siegel \& Castellan, 1988). Analysis of covariance (ANCOVA), Univariable/Multivariable Linear Regression, Poisson Regression (General Linear Regression) were also carried out when relevant (S-PLUS). We first performed univariable analyses to decide which general parameters, such as season, age and breeding status, were significantly related to litter size and pbi. For litter size analysis a Poisson regression model was used, for pbi analysis a linear regression model. Subsequently, the analyses on the effect of NER mutant mice type on litter size and pbi were performed multivariable, such that NER results were corrected for the general effects of season, age or breeding status.

A post-hoc power analysis to detect a twotailed significant difference between NER mutant mice and controls was also carried out, in Win Episcope 2.0. This analysis estimated the minimal number of litters which would be required to detect differences in mean litter size or pbi if the current data were assumed to be from a pilot study. Equality of proportions was tested between expected $\left(\pi_{0}\right.$, according to Mendelian inheritance theory) and observed $\left(\pi_{1}\right)$ number of puppies with genotype $-/-$ from heterozygous couples.

\section{Results}

Age and seasonal effects on reproduction in NER mutant mice

We first analyzed the mouse colony for the effect of parental age and potential seasonal effects on reproductive parameters. When females in heterozygous NER mutant breeding couples did successfully give birth, we observed that litter size was negatively related to age of the male and the female at co-housing $(p=0.02,<0.04$ for male and female, respectively), whereas pbi positively related to age of the male and the female at co-housing $(p=0.001,<0.001$ for male and female, respectively). Heterozygous or homozy- 
Table 4. Mean litter sizes (pups/litter)

\begin{tabular}{|c|c|c|c|c|c|}
\hline \multirow[t]{2}{*}{ Strains/Genotypes } & \multirow[t]{2}{*}{ Number of litters tested } & \multirow[t]{2}{*}{ Mean } & \multirow[t]{2}{*}{ Std. deviation } & \multicolumn{2}{|c|}{$\begin{array}{l}95 \% \text { Confidence interval for } \\
\text { mean from raw data }\end{array}$} \\
\hline & & & & Lower bound & Upper bound \\
\hline Wild type & 13 & $7.0^{\mathrm{a}}$ & 1.5 & 6.3 & 8.0 \\
\hline \multicolumn{6}{|l|}{ Heterozygous couple in pair } \\
\hline CSA & 16 & 5.6 & 1.8 & 4.9 & 6.9 \\
\hline CSB & 16 & $6.9^{\mathrm{c}}$ & 2.1 & 6.0 & 8.3 \\
\hline mHR23B & 18 & $5.1^{\mathrm{b}, \mathrm{d}}$ & 1.6 & 4.2 & 5.7 \\
\hline XPA & 31 & $6.9^{\mathrm{c}}$ & 2.3 & 6.2 & 7.9 \\
\hline XPC & 20 & $6.9^{\mathrm{c}}$ & 2.1 & 6.2 & 8.2 \\
\hline Heterozygous in one gender $\times$ wild type & 39 & 6.1 & 2.3 & 5.3 & 6.8 \\
\hline \multicolumn{6}{|l|}{ Knock out genotypes in pair } \\
\hline KO male & 24 & 6.2 & 1.8 & 5.4 & 7.0 \\
\hline KO female & 13 & 6.7 & 2.1 & 5.5 & 8.1 \\
\hline KO both & 83 & 6.2 & 2.1 & 5.7 & 6.6 \\
\hline
\end{tabular}

Grouping of data according to parental genotype. The values were corrected for age at co-housing of male /female and breeding statuses of male/female based on data collected between 01/01/2001-01/11/2004 (litter size $=0$ was excluded).

${ }^{\mathrm{a}-\mathrm{b}}$ Indicates significant difference between wild type and heterozygous NER couples.

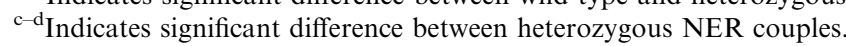

gous knock out NER male/female mice bred at an older ( $>240$ days) age, gave smaller litters and longer pbi than when bred at an age younger than 90 days (data not shown). The breeding of heterozygous and homozygous knock out NER male/ female mice at an older age ( $>240$ days) had a large negative effect on litter size. No significant relations between age of the breeder animals and reproductive performance were found in wild type mice within the same age-range.

Although over a period of 4 years the relatively smallest mean litter size (5.59 pups/litter) was observed when heterozygous couples were cohoused in the summer (June, July, August) and the largest mean litter size (6.14 pups/litter) in autumn (September, October, November), we did not observe significant seasonal differences in either litter size or pbi.

\section{The effect of NER defects on litter size}

We next analyzed the effect of genetic modification of NER genes on litter size (Table 4). Although heterozygous breeding couples of CSB, XPA, XPC, and especially CSA mice $(6.9,6.9,6.9$ and $5.6 \mathrm{pups} /$ litter, respectively), all tend to show smaller litters than wild type breeding couples (7.0 pups/litter), but these differences were not statistically significant. In contrast, heterozygous
mHR23B breeding couples produced significantly smaller litters (5.1 pups/litter $p=0.02)$ when compared to other heterozygous NER couples or wild type parents. We also tested the differences in litter size based on expected phenotypes (couples of otherwise phenotypically normal breeders with a modified genotype vs. $\mathrm{KO}$ in the male, the female or both parental animals); the conclusions remain the same: mHR23B differs.

Breeding couples in which either one or both parents carried a homozygous XPA, XPC, CSA or CSB mutation produced relatively smaller litters, yet not statistically significant, than wild type breeding couples (6.2, 6.7, 6.2 pups/litter for KO in the male, the female or both parental animals, respectively). Since homozygous mHR23B males are sterile and females display reduced fertility $(\mathrm{Ng}$ et al., 2002), our data set does not contain matings with $\mathrm{mHR} 23 \mathrm{~B}-/-$ males and/or females.

Since we observed a clear effect of the mHR23B mutation on the litter size of heterozygous couples, we next investigated whether the reduced number of pups was the result of an overall reduced performance of the heterozygous mHR23B mother, or whether this was due to intrauterine lethality of a particular subgroup of embryos. Only litters with complete data on each individual genotype were available (Table 5). According to Mendelian inheritance theory, heterozygous NER 
Table 5. Expected number of puppies with genotype $-/-$ from heterozygous pairings

\begin{tabular}{llclc}
\hline Strains & Number of litters tested & Number of puppies analyzed & Expected (if Mendelian) & Observed \\
\hline CSA & 16 & 94 & 23.50 & 23 \\
CSB & 15 & 109 & 27.25 & 28 \\
mHR23B & 11 & 53 & $13.25^{\mathrm{a}}$ & $2^{\mathrm{b}}$ \\
XPA & 29 & 203 & 50.75 & 52 \\
XPC & 16 & 108 & 27.00 & 29 \\
\hline
\end{tabular}

${ }^{\mathrm{a}-\mathrm{b}}$ Indicates significant difference from expected.

Table 6. Mean pairing to birth interval, defined as the interval (days) between co-housing the breeder animals and the litter born

\begin{tabular}{|c|c|c|c|c|c|}
\hline \multirow[t]{2}{*}{ Strains/Genotypes } & \multirow[t]{2}{*}{ Number of Litters tested } & \multirow[t]{2}{*}{ Mean pbi } & \multirow[t]{2}{*}{ Std. Deviation } & \multicolumn{2}{|c|}{$\begin{array}{l}95 \% \text { confidence interval for } \\
\text { Mean from raw data }\end{array}$} \\
\hline & & & & Lower Bound & Upper Bound \\
\hline Wild type & 13 & 32.8 & 9.0 & 22.5 & 33.5 \\
\hline \multicolumn{6}{|l|}{ Heterozygous couple in pair } \\
\hline CSA & 16 & $31.5^{\mathrm{a}}$ & 18.5 & 22.7 & 36.4 \\
\hline CSB & 16 & $33.2^{\mathrm{a}}$ & 16.2 & 23.1 & 40.3 \\
\hline MHR23B & 18 & $68.0^{\text {bd }}$ & 48.0 & 53.1 & 100.9 \\
\hline XPA & 31 & $33.7^{\mathrm{a}}$ & 12.8 & 27.8 & 37.2 \\
\hline XPC & 20 & $28.1^{\mathrm{a}}$ & 5.3 & 23.3 & 28.3 \\
\hline Heterozygous in one gender & 39 & $32.4^{\mathrm{c}}$ & 18.5 & 24.9 & 36.9 \\
\hline \multicolumn{6}{|l|}{ Knock out genotypes in pair } \\
\hline KO male & 24 & $32.1^{\mathrm{c}}$ & 18.4 & 23.1 & 38.7 \\
\hline KO female & 13 & 35.9 & 15.9 & 29.3 & 48.5 \\
\hline KO both & 83 & $30.9^{\mathrm{c}}$ & 18.6 & 28.1 & 36.2 \\
\hline
\end{tabular}

Grouping of data according to parental genotype. The values were corrected for age at co-housing of male/female and breeding statuses of male/female based on data collected between 01/01/2001 and 01/11/2004

${ }^{\mathrm{a}-\mathrm{b}}$ Indicates significant difference between heterozygous NER couples .

${ }^{\mathrm{c}-\mathrm{d} I}$ Indicates significant difference between parental genotypes.

couples were expected to have $25 \%$ homozygous knock out (-/-) offspring. However, heterozygous mHR23B couples had a 6.6 -fold $(2 / 13.25)$ significant lower probability of giving birth to knockout $(-/-)$ puppies, while for the other NER mutant strains, homozygous mutant animals were obtained at a Mendelian ratio (Table 5).

\section{The effect of NER defects on pairing to birth} interval (pbi)

A shown in Table 6, we next analyzed the effect of NER mutations on pbi. The heterozygous mHR23B couples displayed a statistically different pbi between parental genotypes $(p=0.001)$. Heterozygous mHR23B couples had a longer pbi
(68.0 days) than matings of any of the other NER mutant genotypes in which parents are heterozygous in only one gender (32.4 days, $p=0.004$ ), $\mathrm{KO}$ in male (32.1 days, $p=0.006)$ and $\mathrm{KO}$ in both (30.9 days, $p=0.001$ ). Significant differences on pbi were also found when comparing heterozygous NER mutations among each other. Also when analyzed per genotype, heterozygous mHR23B couples displayed significantly longer pbi (68.0 days) than all the other heterozygous NER couples $(31.5,33.2,33.7,28.1$ days for heterozygous CSA, CSB, XPA and XPC matings, respectively). Age of male/female at co-housing varied between strains and was positively related to pbi, but after correction for these covariates, the significant difference in pbi was still found $(p<0.01)$. 
Table 7. Power analysis

\begin{tabular}{llll}
\hline Mouse pairings tested & $\begin{array}{l}\text { Number of litters } \\
\text { required per group }\end{array}$ & $\begin{array}{l}\text { Number of litters tested in present } \\
\text { study (NER, comparison group) }\end{array}$ \\
\hline $\begin{array}{l}\text { Litter size } \\
\text { mHR23B heterozygous couples }\end{array}$ & Wild type & 12 & $(18,13)$ \\
XPA heterozygous couples & Wild type & 3536 & $(31,13)$ \\
& KO male & 108 & $(31,8)$ \\
Pairing to birth interval (pbi) & KO both & 126 & $(31,11)$ \\
mHR23B heterozygous couples & Wild type & 4 & $(18,13)$ \\
XPA heterozygous couples & Wild type & 1573 & $(31,13)$ \\
& KO male & 1066 & $(31,8)$ \\
& KO both & 323 & $(31,11)$ \\
\hline
\end{tabular}

Number of litters required per group to detect a two-tailed significant difference between parental genotype if current data are assumed the pilot studies (with $\alpha=5 \%$, power 0.80 ). Data on mHR23B were presented separately because these could not be compared to breeding with KO-parents. XPA was tested selectively since it represents the largest data set on NER-mutant mice and provides thus the best estimate of standard statistical parameters for use in power analysis. KO animals tested in this analysis are XPA KO only.

\section{Discussion}

In the present study, we approached reproductive performance by two hypotheses regarding pregnancy rate: (1) breeding status (first time or multiparous breeder) of male/female animals has an effect on the pregnancy rate and (2) NER mutation has an effect on the pregnancy rate. Generally, multiparous breeders showed a higher pregnancy rate than first time breeders and apparently, NER mutant mice showed the same character in this respect. After correction for age effect, pregnancy rates were still significantly related to breeding statuses (data not shown). The higher pregnancy rate for multiparous breeders might partly be due to the experience of mating and partly to management decisions. Unsuccessful first breeders are eliminated from the breeding program as a management intervention, thus, the multiparous breeders are animals selected on pregnancy success.

An age-dependent decrease in fertility, for example a negative correlation between age and fecundity, has been reported in mice (Shimizu \& Yamada, 2000), and in other species as well (e.g. Osoro \& Wright, 1992). Our epidemiological analysis of the AMS database also uncovered an effect of age on reproductive performance. However, it should be noted that the mice in the present study were between 2 and 9 months of age, and are actually quite young (given a normal lifespan of 1.5-2.5 years in a laboratory context). Age-related sub-fecundity was not expected to turn up so early. We found a trend for negative age effect on litter size in most of the heterozygous NER pairings (CSA, CSB, XPA).

The breeding with heterozygous or homozygous NER mutant mating couples at an older age ( $>240$ days) had a large negative effect on litter size (data not shown), whereas no significant relations were found in wild type mice within the same age-range. This suggests that apart from the known mHR23B defects (discussed below), also other NER mutations might have reduced reproductive performance. In this respect it is interesting to note that Cockayne syndrome is considered a premature ageing syndrome (Bootsma et al., 2001). Whether NER mutations weaken reproductive performance by directly affecting the reproductive organs or indirectly through other physiological interactions justifies more in-depth studies. However, for colony management, some control options are available. A larger litter is expected if males/females are bred at a younger age than 240 days, and for some strains, an even larger litter size is expected if mice were bred at a younger than 90 days.

Heterozygous CS or XP type NER mice, namely CSA, CSB, XPA and XPC in our study, showed smaller litters than wild type mice, especially CSA heterozygous couples (Table 4), but as these differences were not statistically significant, we proposed this was due to either no differences in fact or to the power problem. A post-hoc power 
analysis based on dataset is shown in Table 7. We estimated the minimal number of litters which would be required to detect differences in mean litter size if current data are considered to be pilot studies. The number of pairings used for reproductive differences between genotype to become significant were analyzed for $\alpha=5 \%$ and power 0.8 . The results indicate the number of pairings tested to be adequate for the conclusions drawn. Patients with CS symptoms showed impaired sexual development in some clinical cases (Nance \& Berry, 1992), but no relations and influences of these deficiencies on reproduction in mice have ever been reported up to now. We therefore propose that more physiological data are needed to point out the effect of CS mutation on reproductive performance in mice. Since no information, from literature or otherwise, is available to support the relations between XP mutation and smaller litter size, the mechanisms of XP deficiency on reduced reproductive performance remain to be elucidated.

In marked contrast, litter size was significantly reduced in the case of matings between heterozygous mHR23B mice (5.12 pups/litter). If, in Table 5, the actual number of $2-/-$ mHR23B deficient mice is substituted by the expected 13, mean litter size would amount to 5.8 which is within the range of heterozygous breedings (Table 4). Litters from heterozygous mHR23B parents yielded 6.6-fold lower numbers of homozygous knockout pups than the expected Mendelian $25 \%$. This result is consistent with the initial observation of $\mathrm{Ng}$ and coworkers (2002), reporting that a lack of mHR23B protein causes impaired embryonic development and a higher rate of intrauterine death (likely originating from impaired placental functioning) of mHR23B-/embryos in the heterozygous mother. In addition, homozygous mHR23B pups suffer from perinatal death. The mechanism of intrauterine death, as caused by the mHR23B-/- status is not known and remains open for further study.

We also tested the differences based on expected phenotypes (otherwise phenotype normal couples, excluded wild type couples vs. KO on male, on female and on both); the conclusions however remained the same: heterozygous mHR23B couples produce significantly smaller litters when compared to others. The analyses based on parental genotype (Table 4) and expected phenotype (not shown) were highly consistent, so the latter were not presented separately. The consistency of two tests was due to the absence of many possible genetic combinations. Due to the limitations of breeding decisions, not all possible genetic combinations were included in our analyses or even number of matings in each subgroup (Table 3).

Homozygous mHR23B mice have been reported to display several reproduction-related problems, which were not observed in other NER mutant mice. The problems in homozygous mutant mHR23B males include a reduced number of gonocytes in embryonic testis and a complete absence of spermatogenesis in adult animals. In contrast, homozygous mutant mHR23B females have normally developed ovaries, but when mated with wild type or mHR23B $+/-$ males, produce only one or two pups per litter ( $\mathrm{Ng}$ et al., 2002).

Interestingly, in addition to a smaller litter size, heterozygous mHR23B couples showed a significantly longer mean pbi (68.0 days) than wild type (32.8 days) or heterozygous XP and CS breeding couples. Since mHR23B-/- females show normal mating behavior (as evident from the presence of copulation plugs) and ovaries display the full spectrum of follicular development (e.g. Graafian follicles and corpora lutea), endocrine regulation of ovarian function appears not disturbed, $(\mathrm{Ng}$ et al., 2002). The underlying cause of the lengthened pbi in heterozygous mHR23B pairings remains to be elucidated.

Pairing-to-birth interval in this study was defined as the interval between co-housing the breeder animals and the litter born. The actual dates of mating were not observed or registered. The minimal pbi is expected to range from 20 to 22 days in mice (Festing, 1993). Extended pbi in our study probably reflects extended time needed for a successful mating to occur. In some cases, it might reflect repeated breeding after a pregnancy has broken up. Although an abortion in the third trimester of gestation in mice might have been observed and registered as a clinical event, none were reported.

Gestation length in mice is usually defined as the duration between mating date (observed by detection of a copulation plug) and parturition date. In our study, due to limitations in the data available from AMS, we could only calculate the pbi. Besides, management decisions influenced this dataset. Commonly, if no litter is born within 
8 weeks, the animals will be paired to other partners, but some homozygous knock out mice are less fertile (Cooper et al., 2004) and such couples were allowed to stay together longer. GM strains were thus given more chances to breed than control mice in order to maintain a particular mouse line. The combination of management decisions, natural biological variations and/or the actual effect of NER mutation may have given us a biased measurement on reproductive performance in this study. For example, an unexpected protective effect was found for the relationship between heterozygous or homozygous knock out NER matings and pregnancy rate. It may be safely assumed that reproductive performance (e.g. pregnancy rate) in heterozygous and homozygous knock out NER matings was enhanced by management decisions and did not fully reflect biological differences. More precise observation and study design are necessary to leave out nonbiological effects to elucidate the reason of extended pbi in our study.

Reproductive performance measured in this study is a comprehensive performance including physiological features of both male and female and managerial interventions (as described above). By combining an AMS database with epidemiological analysis, we did reveal some minor phenotypic abnormalities in GM mouse strains and populations which were not immediately evident from the individual mouse through clinical signs. Most strikingly, as known from the literature $(\mathrm{Ng}$ et al., 2002), heterozygous mHR23B couples seemed to be otherwise phenotypically normal, but produce significantly smaller litters. However, our epidemiological approach now also uncovered a longer pbi for heterozygous mHR23B couples compared to wild type and other heterozygous NER mating couples. Without such a systematic database analysis, such subtle problems may go unnoticed. Thus, this study provides a good example on how epidemiological methods, in combination with an Animal Management System, may disclose minor problems as well as support clinical discoveries that may contribute to the generation of hypotheses.

\section{Acknowledgements}

Special grateful to Dr C. Kruitwagen of the Center for Biostatistics, Utrecht University for the assistance of statistical aspects and Sjozef van Baal of the Medical Genetic Centre, Erasmus University Medical Center for his help to extract datasets from AMS. This study was done without animal experimentation, and related protocols were approved by the Animal Experiments Committee under the national Experiments on Animals Act and consistent with the rules laid down in this national law that serves the implementation of 'Guidelines on the protection of experimental animals' by the Council of Europe (1986), Directive 86/609/EC.

\section{Appendix}

The Animal Management System

Fentener van Vlissingen, J.M.

The generation and breeding of GM mice requires a vigorous administrative system to manage the breeding of different genotypes and to maintain information on pedigree, genotype and phenotype of the animals. Ethical considerations and legal requirements (European Union, 1986) prompted the in house development of an integrated Animal Management System (AMS) that also allows for the registration of clinical observations and the management of the health and welfare of animals on experiment as well as breeding or experimental stock (Van Baal \& Verkerk, 2005). There is a record for each animal being identified by a unique number. Also, each animal is marked and accompanied by a bar-code label that is read for every mutation or addition of data in the animal room (Figure 1). The animal ID is fixed to its permanent data (parents, date of birth, sex) and linked to non-permanent information such as room number, cage number, transponder number, project, investigator, et cetera. The system was designed to manage a colony including hundreds of genotypes, between 10,000 and 20,000 litters and some 40,000 animals on test annually and is a web-based mySq1 database that can be accessed by authorized persons through the network. It is used jointly by investigators, animal technicians and animal care personnel, the management of the animal facility and internal officials such as the animal welfare officers. Users are defined by name, department, group and specific rights. Feedback information on clinical signs is recorded by the animal caretakers and the severity and duration of 


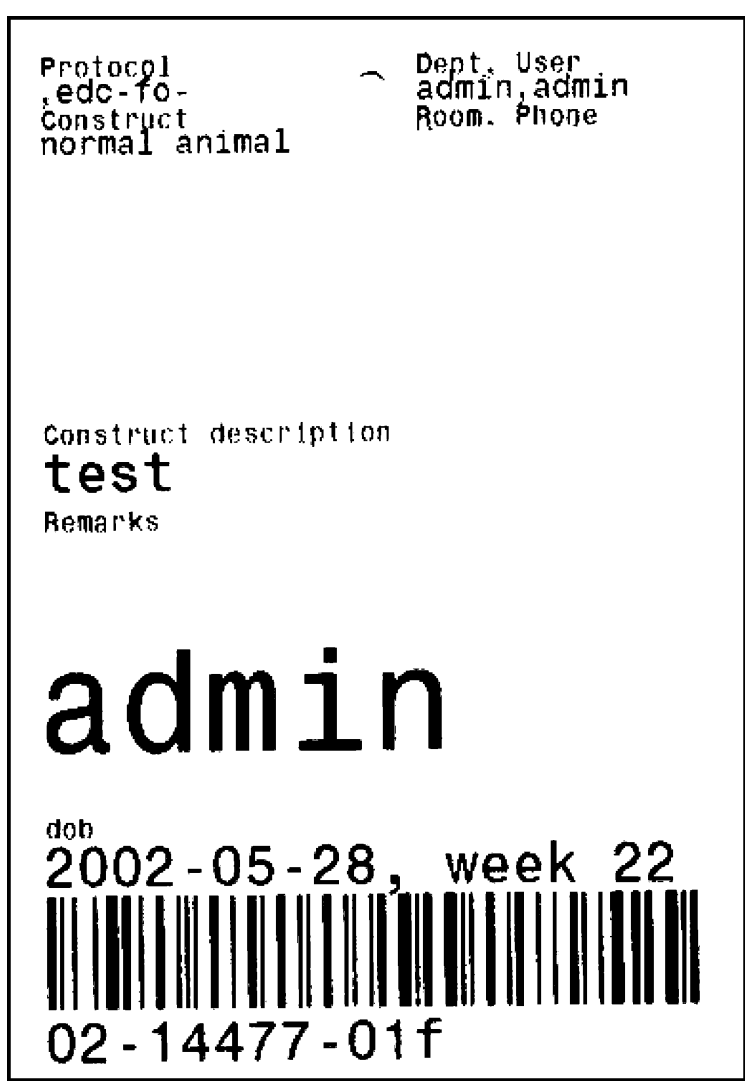

Figure 1. Example of the cage label of one animal identified by a unique number composed of year of birth, litter number, number within the litter, and a unique barcode.

the signs combined generate alerts (Figure 2). The principles of humane endpoints are applied in a systematic manner to prevent any more discomfort than is inevitable to complete the experiment (Fentener van Vlissingen et al, 1999, OECD, 2000). Four main categories of clinical observations were defined: general appearance, spontaneous behavior, response to stimuli, and body weight. The default value is zero, any deviation is graded $1-3$, and the investigator is alerted when an animal scores a total of 3 or more. The score is downgraded when the condition of the animal improves, but upgraded when the deviations remain the same over several days or aggravate. In the absence of a specific justification to observe the further developments, the animal is killed at the indication of a grade of more than 3 in total. Both instructions to kill animals and breeding instructions result in listed daily work instructions for each animal room. To avoid agedependent pathology, the system monitors mice not to be bred when older than 6 months of age and to be killed humanely when they reach an age of 8 months, unless the ethical committee has allowed aging as part of the project This is one of many options that can be defined by selection from a menu (Figure 3). As a result, mice are typically bred several times when young, and one more time at the age of 6 months to lengthen the generation interval for the reduction of putative genetic drift. Male and female(s) are bred at the specific instruction of the investigator by entering the desired breeding combination in the system by animal numbers (Figure 4). Timed mating of the female can be registered following the optional instruction to check daily for the presence of a copulation plug, followed by separation of the breeding pair. The system will then generate the expected date of birth. This option is not used routinely. Birth of litters is registered on a daily base throughout the colony. Each litter is assigned a litter number and both dead and alive newborns are counted and registered by a matching series of individual ID-numbers and by sex (or: sex

\begin{tabular}{|c|c|c|c|c|c|c|c|c|c|c|}
\hline animalid & construct & genotype & sex & dob & Age(w) alive & in breeding & allow aging & stable & protocol & remarks \\
\hline $01-1228-00$ & & wt & $\mathrm{m}$ & 2001-01-02 & $82,6[x]$ & [] & aging & C06 & $132-00-32$ & $+1-x+1-$ \\
\hline 01-1228-02 & & wt & $\mathrm{m}$ & 2001-01-02 & $82,6[]$ & {$[x]$} & aging & $\mathrm{C06}$ & $132-00-32$ & $+1-x+1-$ \\
\hline \multicolumn{11}{|c|}{ 01-1228-03 Appearance: 0 Behaviour: 0 Response } \\
\hline $01-1228-03$ & & wt & $m$ & 2001-01-02 & $82,6[x]$ & [ ] & aging & $\mathrm{CO6}$ & $132-00-32$ & $+1-x+1-$ \\
\hline
\end{tabular}

Figure 2. Section of a report (data exported from database to spreadsheet) indicating that animal \# 01-1228-03 was found dead (and by whom), before the animal was declared dead in the database, yet (otherwise, if declared dead in the database, it would not appear in this listing, just like littermates 04, 05, 06 and 07 that were deleted from this listing but kept in the historic part of the database). Another animal born in the same litter (\# 01-1228-08) received a score on behavior and a remark on general condition (fat). 


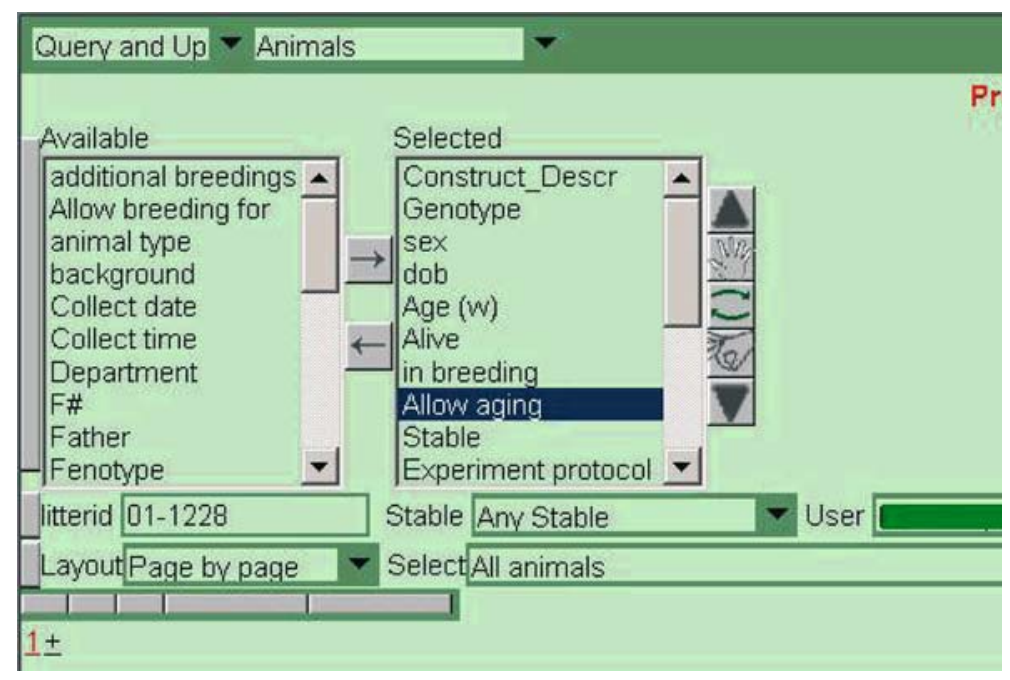

Figure 3. Section of a screen indicating some options to be used in queries for listing animals according to the users preferences. The selection in this case is litter \# 01-1228, the same as presented in Figure 2.

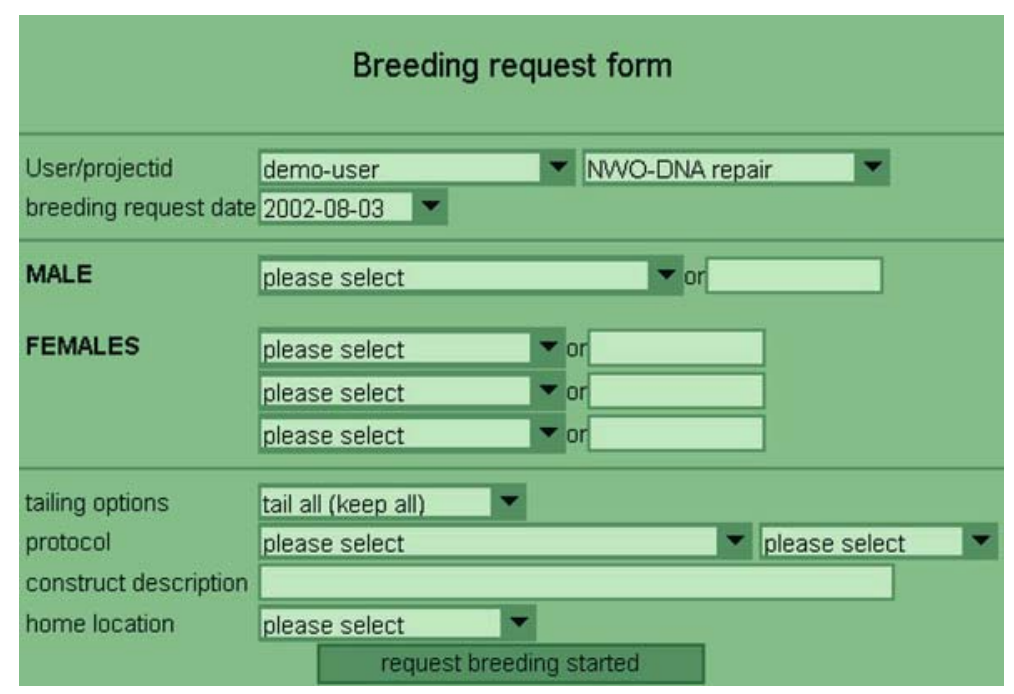

Figure 4. Breeding request form (screen) showing options to order specific animals to be co-housed for breeding. Investigators authorized for a specific project can use this to order a breeding of animals assigned to that project, using his own network connection. Instructions like this one will then be listed for use by the animal caretakers who will register the execution of the work in AMS by date.

unknown). Subsequently, any puppies that die or are killed for humane reasons are sexed (if possible) and registered by date and circumstances of death. Genotyping is done using tissue biopsies taken from the tip of the tail at approximately 10 days of age. At this time, the animals are identified by toe marks. Any culling of puppies (e.g. homozygous wild type offspring resulting from heterozygous breeding) is done at the time of weaning and linked to the individual animals.
The system was made available to other institutions (http://www.eur.nl/fgg/ch1/ams)

\section{References}

Altman DG (1991) Comparing groups - continuous data. In: Altman DG (ed), Practical Statistics for Medical Research. Chapter 9. (pp. 179-228) Chapman \& Hall/CRC, London.

Amiram R and Glickman BW (2001) Human DNA Repair Genes. Environ Mol Mutagen 37: 241-243. 
Baal JBJM van and Verkerk A, The Animal Management System (AMS). Web site http://www.eur.nl/fgg/ch1/ams (lower case) Rotterdam, The Netherlands.

Baarends WM, Roest HP and Grootegoed JA (1999) The ubiquitin system in gametogenesis. Mol Cell Endocrinol 151: $5-16$.

Berneburg M and Krutmann J (2003) Xeroderma pigmentosum and related Syndromes. Hautarzt 54: 33-40.

Beynen AC and Hau J (2001) Animal models. In: van Zutphen LFM, Baumans V and Beynen AC (eds), Principles of Laboratory Animal Science. (pp. 197-205) Elsevier, Amsterdam.

de Boer J, Andressoo JO, de Wit J, Huijmans J, Beems RB, van Steeg $\mathrm{H}$ et al. (2002) Premature aging in mice deficient in DNA repair and transcription. Science 296: 1276-1279.

de Boer J and Hoeijmakers JHJ (1999) Cancer from the outside, aging from the inside: mouse models to study the consequences of defective nucleotide excision repair. Biochimie 81: 127-137.

de Boer J and Hoeijmakers JHJ (2000) Nucleotide excision repair and human syndromes. Carcinogenesis 21: 453-460.

Bootsma D, Kraemer KH, Cleaver JE and Hoeijmakers JHJ (2001) Nucleotide excision repair syndromes: Xeroderma pigmentosum, Cockayne syndrome and trichothiodystrophy. In: Scriver CR, Beaudet AL, Sly WS and Valle D (eds), The Metabolic and Molecular Bases of Inherited Disease. vol. 1. (pp. 677-703) McGraw-Hill Book Co, New York.

Boshier DP (1968) The relationship between genotype and reproductive performance before parturition in mice. $J$ Reprod Fertil 15: 427-435.

Cheo DL, Meira LB, Hammer RE, Burns DK, Doughty ATB and Friedberget EC (1996) Synergistic interactions between $\mathrm{XPC}$ and p53 mutations in double-mutant mice: neural tube abnormalities and accelerated UV radiation-induced skin cancer. Curr Biol 6: 1691-94.

Cooper TG., Yeung CH, Wagenfeld A, Nieschlag E, Poutanen M, Huhtaniemi I et al. (2004) Mouse models of infertility due to swollen spermatozoa. Mol Cell Endocrinol 216: 55-63.

European Union (1986) Directive 86/ 609/EEC on the protection of animals used for experimental and other scientific purposes. Web site http://europa.eu.int.

Fentener van Vlissingen JM, Beems RB and van Dijk EJ (1999) Retrospective evaluation of clinical signs, pathology and related discomfort in chronic studies. In: Hendriksen CFM and Morton DB (eds), Humane Endpoints in Animal Experiments for Biomedical Research. (pp. 89-94) Laboratory Animals, London.

Festing MFW (1993) Origins and characteristics of inbred strains of mice. 11th listing. Mouse Genome 91: 393-550.

Grootegoed JA, Baarends WM, Roest HP and Hoeijmakers JHJ (1998) Knockout mouse model and gametogenic failure. Mol Cell Endocrinol 145: 161-166.

Hogan B, Beddington R, Costantini F and Lacy E (1994) Developmental genetics and embryology of the mouse: Past, present, and future. In: Hogan B, Beddington R, Constantini F and Lacy E (eds), Manipulating the Mouse Embryo - A Laboratory Manual. (pp. 1-18) Cold Spring Harbor Laboratory Press, Cold Spring Harbor.

van der Horst GTJ, van Steeg HBR, van Gool AJ, de Wit J, Weeda G, Morreau H, Beems RB et al. (1997) Defective transcription-coupled repair in Cockayne syndrome B mice is associated with skin cancer predisposition. Cell 89: 425-435.

van der Horst GTJ, Meira LB, Gorgels TGMF, de Wit JSVM, Richardson JA, Kamp Y et al. (2002) UVB radiation-induced cancer predisposition in Cockayne syndrome group A (Csa) mutant mice. DNA Repair 1: 143-157.

Lehmann A (2002) Ageing: repair and transcription keep us from premature ageing. Curr Biol 12: 550-551.

Lyon MF and Searle AG (1989) Strains of the laboratory mouse. In: Lyon MF and Searle AG (eds), Genetic Variants and Strains of the Laboratory Mouse, 2nd edn. Oxford University Press, Oxford.

van der Meer M, Baumans V, Frans MAH, Olivier B and van Zutphen BLM (2001) Consequences of gene targeting procedures for behavioral responses and morphological development of newborn mice. Trans Res 10: 399-408.

Nance MA and Berry SA (1992) Cockayne syndrom: review of 140 cases. Am J Med Genet 42: 68-84.

Ng JM, Vrieling H, Sugasawa K, Ooms MP, Grootegoed JA, Vreeburg JT, Visser P et al. (2002) Developmental defects and male sterility in mice lacking the ubiquitin-like DNA repair gene mHR23B. Mol Cell Biol 22: 1233-1245.

OECD (1998) Why Mice Matter: Novel Systems for the Study of Human Disease: From Basic Research to Applications The Rome 96 Work shop, Paris.

OECD (2000) Guidance Document on the Recognition, Assessment, and use of Clinical Signs as Humane Endpoints for Experimental Animals Used in Safety Evaluation. OECD Environmental Health and Safety Publications, Series on Testing and assessment, 19 OECD, Paris, France, $39 \mathrm{pp}$.

Osoro K and Wright IA (1992) The effect of body condition, live weight, breed, age, calf performance, and calving date on reproductive performance of spring-calving beef cows. $J$ Anim Sci 70: 1661-1666.

Palmiter RD, Brinster RL, Hammer RE, Trumbauer ME, Rosenfeld MG, Birnberg NC et al. (1982) Dramatic growth of mice that develop from eggs mircoinjected with metallothionein-growth hormone infusion genes. Nature 300: 611615

Peters AG, Bywater PM and Festing MF (2002) The effect of daily disturbance on the breeding performance of mice. $L a b$ Anim 36: 188-192.

Shimizu K and Yamada J (2000) Relationship of decrease in fecundity with advancing age to structural changes in mouse endometrium. $J$ Anat 196: 111-114.

Siegel S and Castellan NJ Jr (1988) The case of k independent samples. In: Siegel S and Castellan NJ (eds), Non-parametric Statistics for the Behavioral Sciences. vol. 1. (pp. 190-222), 2nd edn. McGraw-Hill, New York.

Simpson ER (1998) Genetic mutations resulting in estrogen insufficiency in the male. Mol Cell Endocrinol 145: 55-59.

de Vries A, van Oostrom CT, Hofhuis FM, Dortant PM, Berg RJ, de Gruijl FR et al. (1995) Increased susceptibility to ultraviolet-B and carcinogens of mice lacking the DNA excision repair gene XPA. Nature 377: 169-73.

van Zutphen LFM, Hedrich HJ, Van Lith HA and Prins JB (2001) Genetic standardization. In: van Zutphen LFM, Baumans V and Beynen AC (eds), Principles of Laboratory Animal Science. (pp. 129-147) Elsevier, Amsterdam. 